\title{
Improved Reliability and Sensitivity for Priming DNA Sequences with Contiguous Strings of Hexamers
}

\author{
Jan Kieleczawa1), Carl W. Fuller2) and F. William Studier1)
}

1) Brookhaven National Laboratory, Biology Department, Bldg. 463, Upton, N.Y. 11973; 2) Amersham Life Science Corporation, 26111 Miles Road, Cleveland, $\mathrm{OH} 44128$

\section{Introduction}

Efficient DNA sequencing is a necessity for the large-scale projects now being proposed. Efficiency, of course, may have a different meaning depending on the availability of materials used for sequencing. One common approach to sequencing large template DNAs is to randomly sequence small fragments until the entire sequence is covered. This approach is simple and effective, and is appropriate if more directed strategies are too slow or require materials that are difficult to obtain. The random approach, however, ensures that most of the sequence will be determined repeatedly. Another approach is to "walk" along a sequence with custom-made primers. This approach is very efficient since the sequence of very few bases is determined more than once. It is, however slow and expensive since custom primers are expensive and take 1-2 days to synthesize. Once made, only a small fraction of the primer is actually used.

An approach which takes advantage of the high efficiency of primer walking but avoids the time and expense of custom primer synthesis was described recently by kieleczawa et.al. (1). They discovered that specific sequence priming can be obtained using three or more contiguous 6-mer oligonucleotides (hexamers). The specificity of priming approaches that of 18-mer primers. Since there are only 4096 possible hexamers, the entire collection of 4096 primers can be synthesized, divided and srranros in $\rightarrow$ 
library, so that many laboratories can share them. In this way, custom synthesis of 18-mer or larger primers is avoided. For a given step in the primer walk, a priming sequence of 18 bases (spanning 3 adjacent hexamer sequences) is chosen, the appropriate 3 hexamers from the collection of 4096 are taken and sequencing performed directly. No delay for primer synthesis is experienced, and the cost of individual primers is greatly reduced since hexamers will be re-used in many different combinations.

This method takes advantage of a property of the single-stranded DNA binding protein (SSB) of E. coli. When the mixture of three hexamers is added to a DNA template to be sequenced, the primers ordinarily will prime synthesis at many sites individually as well as at the specific, contiguous site intended. When SSB protein is added and the mixture incubated at $0^{0} \mathrm{C}$ priming is specific, presumably because the binding protein displaces single hexamers from the template but not strings of 3 or more contiguous hexamers. These stable strings of hexamers serve as primers for T7 DNA polymerase in sequencing reactions.

This primer-walking method has great potential for improving the efficiency of DNA sequencing. However, there are several areas where additional improvements can be made. Improvements in general reliability and robustness of the method will be beneficial for making the method more generally useful in all laboratories. This work was focused on one area where a fundamental improvement could be made to the method. Specifically, we concentrated our efforts on testing different DNA binding proteins for the ability to promote specific priming with strings of hexamers and to minimize the resolution-reducing effects these proteins have on electrophoretic separations.

\section{Materials \& Methods}

All E. Coli SSB mutants and other DNA-binding proteins were obtained from J. Chase (2), of Amersham Life Sciences. These are listed in Table $I$. Several mutants of $E$. coli SSB protein were used. The description of a particular mutant refers to an amino 
acid substitution at a given position. For example SSB Ala-60 is a mutant where Phenylalanine at position 60 was replaced by Alanine. Figure 1 shows amino acid and DNA sequences of $E$. coli SSB highlighting the mutations.

Single stranded DNA from bacteriophage M13 was purified according to a standard molecular biology methods (3). Source and quality of all chemicals were as in (1). DNA sequencing with $35 \mathrm{~S}-$ dATP detection method were as described in (1). Purity and integrity of proteins were checked by polyacrylamide gel electrophoresis (15\% acrylamide, $0.1 \%$ SDS) according to Laemmi (4).

\section{Results}

\section{Effect of various DNA-binding proteins on DNA sequencing primed with strings of hexamers.}

All DNA-binding proteins were individually tested for the ability to promote specific priming of the sequencing reaction on M13 DNA by string of three hexamers with 3' at nucleotide 1165 (AAGCGC, GAAACA, AAGTAC). All labeling-step reactions had a volume of $10 \mathrm{Hl}$ and contained the different amount of binding protein. Without SSB or with less than $2 \mu \mathrm{g}$ of protein in the reaction mixture (for wild type SSB) priming is weak and nonspecific. With more than $7.5 \mu \mathrm{g}$, under the same reaction conditions, SSB is inhibitory and signal strength is diminished. Thus, there is a limited range of protein concentration where results are optimal (1). We titrated sequencing reactions with DNA binding proteins in the range $0-10 \mu \mathrm{g}$ in a standard reaction mixture.

Of all the SSB mutants tested, only SSB Cys-87 did not stimulate hexamer-string priming of DNA sequences. All other mutants promoted priming. The optimal amounts for each protein differed but is in the range of $1.5-3 \mu \mathrm{g} /$ reaction. Furthermore $\mathrm{gp} 32$ and gp2.5, DNA-binding proteins from bacteriophages $\mathrm{T} 4$ and $\mathrm{T} 7$ were 
also able to stimulate DNA sequencing primed with hexamer strings. on the other hand, DNA-binding proteins isolated from prokaryotic transmissible plasmids plP71a, plP231a and $E$. coli F sex factor were unable to promote hexamer-string priming. At the moment we do not have an explanation why these proteins fail to stimulate DNA sequencing. To eliminate possibility that these proteins may be degraded and therefore unable to bind to DNA, an analysis by SDS gel electrophoresis of all the DNA binding proteins was carried out. The gel indicated that SSB CYS-87 is intact so failure to stimulate DNA sequencing may suggest the importance of Lys 87 at this position in E. Coli SSB. For example, the recently reported crystal structure of gp32 indicates that an analogous amino acid, Lys 110 makes an electrostatic contact with 3' phosphate oxygens (5).

\section{Removal of SSBs from sequencing reactions.}

DNA binding proteins, including $\mathrm{SSB}$, can reduce the resolution of the polyacrylamide gels used for DNA sequencing. The proteins can apparently bind DNA even in the presence of urea or other denaturants causing band smearing. Thus, SSB must be dissociated from DNA prior to electrophoresis. Satisfactory results using radiolabeled sequencing methods are obtained by including $0.25 \%$ SDS in stop mixture (1). However, this treatment is not sufficient (this work, unpublished) when sequencing is done using fluorescent dye-terminators with electrophoresis on the ABI 373 a automated DNA sequencing instrument. One effective but cumbersome method to remove SSB is by extraction with phenol and chloroform (6). A simpler and more readily automated procedure to remove interference of SSB is to digest the reaction products with the proteolytic enzyme pronase followed by ethanol precipitation (6).

In this work we took advantage of the properties of the SSB-1 mutant ( His-55 is replaced by Tyr-55). This mutant protein is not as effective in helix-destabilizing as wild-type SSB when measured by its ability to decrease the thermal melting transition of DNA 
(7). However, it is just as effective in stimulating hexamer priming of sequencing reactions as wild-type SSB. We have found that neither extraction nor digestion is required in order to obtain sharp electrophoresis bands when SSB-1 mutant is used for hexamer string primed sequencing.

\section{Discussion}

We have tested several E.Coli SSB mutants and some other DNAbinding proteins for the ability to stimulate hexamer primed DNA sequencing. With the exception of SSB Cys-87, all E. Coli SSB mutants produced comparable signal quality and strength. Lack of stimulation by SSB Cys-87 may indicate that Lys-87 in wild-type protein is critical to the DNA-protein interaction. When the crystal structure of SSB is solved, the amino acid residues responsible for interaction with DNA should be identified so that more rational site-directed mutants can be designed and constructed with desired properties.

The most important result of this work was finding that SSB-1 mutant does not require any post-reaction treatment in order to render clear and strong band patterns on the sequencing electrophoresis gels. This not only simplifies sequencing process but may prove to be of crucial importance for automation of hexamer primed DNA sequencing. Complete removal of interference by SSB may also be critical for capillary-based sequencing methods. Further work in this direction is currently in progress.

\section{References}

1. Kieleczawa, J., J.J. Dunn and F.W. Studier. 1992. DNA Sequencing by Primer Walking with Strings of Contiguous hexamers. science 258:1787-1791.

2. Ruvolo, P.P., K.M. Keating, K.R. Williams and J.W. Chase. 1991. Single-Stranded DNA Binding Proteins (SSBS) from Prokaryotic Transmissible Plasmids. PROTEINS: structure, Function and Genetics $9: 120-134$. 
3. Sambrook, J., E.F. Fritsch and T. Maniatis. 1989. In Molecular Cloning. A Laboratory Manual. Cold Spring Harbor Laboratory Press. Cold Spring Harbor. New York.

4. Laemmli, U.K. 1970. Cleavage of Structural Proteins during the Assembly of the Head of Bacteriophage T4. Nature 227:680-685.

5. Shamoo, Y., A.M. Friedman, M.R. Parsons, W.H. Konigsberg and T.A. Steitz. 1995. Crystal Structure of a Replication Fork SingleStranded DNA Binding Protein ( $T 4$ gp 32 ) Complexed to DNA. Nature $376: 362-366$.

6. McCombie, W.R. and J. Kieleczawa. 1994. Automated DNA Sequencing Using 4-Color Fluorescent Detection of Reactions Primed with Hexamer Strings. BioTechniques 17:574-579.

7. Williams, K.R., J.B. Murphy and J.W. Chase. 1984. Characterization of the structural and Functional Defect in the Echerichia coli Single-Stranded DNA Binding Protein Encoded by the ssb-1 Mutant Gene. J.Biol.Chem. 259:11804-11811. 
TABLE 1. List of Single-Stranded DNA Binding Proteins Used in This Work.

$\begin{aligned} \text { No. } & \text { DNA Binding } \\ \text { 1. } & \text { SSBwt } \\ \text { 2. } & \text { SSB-1 } \\ \text { 3. } & \text { SSB-113 } \\ \text { 4. } & \text { SSB Ala-60 } \\ \text { 5. } & \text { SSB Phe-40 } \\ \text { 6. } & \text { SSB Phe-54 } \\ \text { 7. } & \text { SSB Phe-135 } \\ \text { 8. } & \text { SSB Cys }-70 \\ \text { 9. } & \text { SSB Cys-87 } \\ \text { 10. } & \text { SSB Leu-55 } \\ \text { 11. } & \text { FSSB } \\ \text { 12. } & \text { gp2.5 } \\ \text { 13. } & \text { gp32 } \\ \text { 14. } & \text { p1P71a } \\ \text { 15. } & \text { plP231a }\end{aligned}$

Comments

wild type

His -55 - Tyr -55

Pro-176 - Ser -176

Phe-60 - Ala-60

Trp-40 - Phe -40

Trp-54 - Phe-54

Trp-135 - Phe-135

Tyr-70 - Cys -70

Lys-87 - Cys-87

His-55 - Leu-55

from E.Cōli F sex factor

from bacteriophage $\mathrm{T} 7$

from bacteriophage $\mathrm{T} 4$

pBR322+ECORI DNA fragment of plP71a, which encodes plp7la DNA binding protein. pBR322+ECORI DNA fragment of plP231a, which encodes plP231a. DNA binding protein. 
FIGURE 1. Single-Stranded DNA Binding Mutations Used in This Work.

$118 / 0$

atg gcc agc aga ggc gta aac aag gtt att ctc gtt ggt aat ctg ggt cag gac ccg gaa Met ala ser arg gly val asn lys val ile leu val gly asn leu gly gln asp pro glu

$178 / 20$

gta cgc tac atg cca aat ggt ggc gca gtt gcc aac att acg ctg gct act tcc gaa tca val arg tyr met pro asn gly gly ala val ala asn ile thr leu ala thr ser glu ser

$238 / 40$

tgg cgt gat aaa gcg acc ggc gag atg aaa gaa cag act gaa tgg cac cgc gtt gtg ctg TRP arg asp lys ala thr gly glu met lys glu gln thr glu TRP HIs arg val val leu PHE

ssb phe -40 TYR

ssb-1

PHE

ssb phe-54

LEU

ssb leu-55

$298 / 60$

ttc ggc aaa ctg gca gaa gtg gcg agc gaa tat ctg cgt aaa ggt tct cag gtt tat atc PHE gly lys leu ala glu val ala ser glu tyr leu arg lys gly ser gln val tyr ile ALA

$\mathrm{ssb}$ ala -60 CYS $\mathrm{ssb}$ cys -70

$358 / 80$

gaa ggt cag ctg cgt acc cgt aaa tgg acc gat caa tcc ggt cag gat cgc tac acc aca glu gly gln leu arg thr arg LYS trp thr asp gln ser gly gln asp arg tyr thr thr CYS

ssb cys -87

$418 / 100$

gaa gtc gtg gtg aac gtt ggc ggc acc atg cag atg ctg ggt ggt cgt cag ggt ggt ggc glu val val val asn val gly gly thr met gln met leu gly gly arg gln gly gly gly

$478 / 120$

gct ccg gca ggt ggc aat atc ggt ggt ggt cag ccg cag agc ggt tgg ggt cag cct cag ala pro ala gly gly asn ile gly gly gly gln pro gln ser gly trp gly gln pro gln PHE

ssb phe -135

$538 / 140$

cag cog cag ggt ggc at cag ttc agc ggc ggc gcg cag tct cgc ccg cag cag tcc gct gln pro gln gly gly asn gln phe ser gly gly ala gln ser arg pro gln gln ser ala

$598 / 160$

ccg gca gcg ccg tct aac gag ccg ccg atg gac ttt gat gat gac att ccg ttc tga pro ala ala pro ser asn glu pro pro met asp phe asp asp asp ile PRO phe OPA

SER

$\mathrm{SSB}-113$ 


\begin{abstract}
Efficient DNA sequencing is a necessity for the large-scale projects now being proposed. One strategy is to "walk" along a sequence with custom-made primers. This approach is very efficient since the sequence of very few bases is determined more than once. It is, however slow and expensive since custom primers are expensive and take 1-2 days to synthesize. Once made, only a small fraction of the primer is actually used. A potential solution to these problems was described recently (Kieleczawa, Dunn and Studier Science 258:1787-1791, 1992). They discovered that specific sequence priming can be obtained using three or more contiguous 6mer oligonucleotides in the presence of a single-stranded DNA binding protein (ssb). Since there are only 4096 possible sequences for 6 nucleotide DNAs, the entire collection of 4096 primers can be synthesized and divided up so that many laboratories can share them. No delay for synthesis is experienced, and the cost of individual primers is greatly reduced since hexamers will be re-used.

We have explored several aspects of primer walking using hexamers, and have tested a number of ssb proteins for hexamer primed sequencing. We found that some ssb proteins do not support hexamer primed sequencing while others have specific advantages. One mutant of $E$. coli ssb (ssb-1) is readily inactivated and thus has specific advantages for hexamer-primed sequencing.
\end{abstract}

